M IIIajur purpuse UI rile I ecnnical Information Center is to provide the broadest dissemination possible of information contained in DOE's Research and Development Reports to business, industry, the academic community, and federal, state and local governments.

Although a small portion of this report is not reproducible, it is being made available to expedite the availability of information on the research discussed herein. 
TITLE MORE ON THE FIRST ORDER CHIRAL SYMMETRY

AUTHOR(S): Rajan Gupta

SUBmited to Proceedings of the 1986 Brookhaven Conference on Lattice Gauge Theory

\title{
DISCI.AIMER
}

\begin{abstract}
This report was prepured as un account of work sponsmreal hy an hgency of the United Sitates (iovernment. Neither the United Siates Ciuvernment nor uny upency thereof, nur hlly of their employeen, mukes uny wurranly, express ur implied, of usoumes nny legul liability of responai. hility for the accuracy. completeness, of uscfulness of any informution, appariutur, proxfuct, of proceen disclowed, or reprenents that its use wosuld not infringe privutely owned rights. Refer. unce herein to any speciffe commercial proxluci, proxesa, or service hy Irade numr, Irudemarh, manufacturor, or otherwine doen not necessarily conntitute or imply its endorsement, reconnmendation, of favoring by the United Situlen (tovernment or any agency theresf. The views and opinions of authors expressed herein do not necesurily state or reflect thase of the United States Government or any asency thereof.
\end{abstract}




\title{
MORE ON THE FIRST ORDER CHIRAL SYMMETRY
}

\section{TRANSITION IN QCD}

\author{
Rajan Gupta \\ J. Robert Oppenheimer Fellow, \\ Theoretical Division, MS-B285, \\ Los Alamos National Laboratory,Los Alamos, N.M 87545.
}

\begin{abstract}
We present evidence from numerical simulations for a first order chiral symmetry restoration tzansition in QCD at finite temperature. Also, for quarks in the fundamental representation, there is a simultaneous deconfinement transition. These transitions are present only for small quark masses. We use an exact algorithm to incorporate the dynamical quarks.
\end{abstract}

In [1] we presented evidence for the chiral symmetry restoration and deconfinement transition at $6 / g^{2}=4.9$ and $m_{q}=0.025$. In this talk I shall present results at $6 / g^{2}=4.95$. The work was done in collaboration with $G$. Guralnik, G. Kilcup, A. Patel and S. Sharpe at Los Alamos.

We are pursuing a program aimed at developing an algorithm efficient at small $g$ and $m_{q}$. In particular we want to investigate how and when systematic biases become dominant in various approximate algorithms. To do thig we have made extensive runs using the exact algorithm of Scalapino and Sugar [2| to act as a standard for comparison. The largest lattice on which this can be done with reasonable statistics is $4 \times 4 \times 4 \times 4$. In exploring the small quark mass limit we find evidence for the chiral transitiun. In this talk I will concentrate on this physics result. Due to the small lattice size, $4^{4}$, and the fact that $N_{\text {apatial }}=N_{\text {lime }}$, we do not claim any quantitative results. Clearly, in a roalistic finite temperature study, the transition temperature $w_{1} l l$ be shifted. However, we think that the clarity of the signal of the transition in our data, together with our use of an exact algorithm, make our qualitative result intercsting.

Simulations of pure gnuge SU(3) show a strong first order transition at a temperature $T_{c} \approx \Lambda_{\text {MS }}$ [3|. A: this transition the global $Z(3)$ symmetry of the theory is spontaneously broken. A non-zero expectation value of the Polyakov line $\langle L\rangle$ in the high temperature deconfined phast: implies a finite free energy for the quarks. A second order parameter, the chiral condensate 
$\bar{\chi} \chi$ measured in the quenched approximation, is also discontinuous at the transition. $\bar{\chi} \chi$, when extrapolated to $m_{q}=0$, changes from a non-zero value at low $T$ to zero in the high $T$ phase.

Dynamical quarks act as extemal fields and explicitiy break the $Z(3)$ symmetry. $\langle L\rangle$ is still a measure of the quark free energy but it is non-zero for all temperatures due to vacuum polarization. $\bar{\chi} \chi$ remains a good order parameter to study chiral symmetry. The only theoretical understanding of the realization of chiral symmetry comes from a renormalization group analysis of an effective spin model in $4-\epsilon$ dimensions [4]. The conclusion is that QCD has a first order chiral symmetry transition for $N_{f} \geq 3$ and at $m_{q}=0$. For $T<T_{c}$, one expects $\bar{\chi} \chi \neq 0$ when extrapolated to $m_{q}=0$. For $T>T_{c}$ the chiral symmetry is restored, consequently $\bar{\chi} \chi \propto m_{q}$ for small $m_{q}$. This needs to be verified. Also, if, as in the pure gauge theory, there is a discontinuity in $\langle L\rangle$, then we expect to see interesting thermodynamical properties of the quark-gluon plasina [5] created in heavy ion collisions.

The expected phase diagram foi $Q C D$ is as follows: The confinemenc transition at $m_{q}=\infty$ extends to some finite $m_{q}$ in the $m_{q}-T$ phase plane, and similarly the chiral transition at $m_{q}=0$ extends to some non-zero $m_{q}$. The questions to settle are whether these two transitions are connected and whether the chiral transition with the three physical light flavors is first order. The status of the rhiral transition is not clear. We summarize the results for 4 flavors of staggered fermions obtained using approximate algorithms. The most detailed calculations are by Kogut et al. [G] who find a a apid crossover for $m_{q}=0.1$ and 0.05 . They extrapole the senter of the cross-over to $m_{q}=0$, and th ireby estimate the transition coupling $6 / g^{2}$ for $N_{t}=6(4)$ to be $5.01 \pm 0.025(\approx 4.9)$. Further, assuming that asymptotic scaling is valid, they estimate the transition temperature to be $T_{c}=(2.14 \pm 0.1) \Lambda_{\overline{M S}}$. These results are obtained with both the micro-canonical and the hybrid algorithm. Similarly, Gavai, $[\gamma \mid$ does not find evidence for .c first order transition using the pseudo-fermion algorithm. On the other hand, Fucito and Solomon [8] claim to see a first order transition at $m_{q}=0.1$. They used perturbation theory to reduce the rumber of flavors to 3 . Fiskugita and Ukawa. [9] use the Langevin algorithm and also claim that at $m_{q}=0.1$ the transitior is already first order. The chicf criticism against the last two calcularions which found a hysteresis, i.e. two meiastable states, has been that the runs were not long enough for complete the:malization. Also, the algorithm used to simulate fermions is different in eais study so the reason for conflicting results ma:" lie in the nature of the bias introduced due to the approximation.

In the exact algorithm the ratio of determinants $R \equiv \operatorname{det}(1+$ $\left.M^{-1} \delta M\right)$ is calculated at each link update. Since we use staggered formions (4 fivors), the algorithm requires a calculation of a $6 \times 6$ bluck of $M^{-1}$. Because $M I^{-1}$ is calculated with the conjugate gradient (CG) iterative algo- 


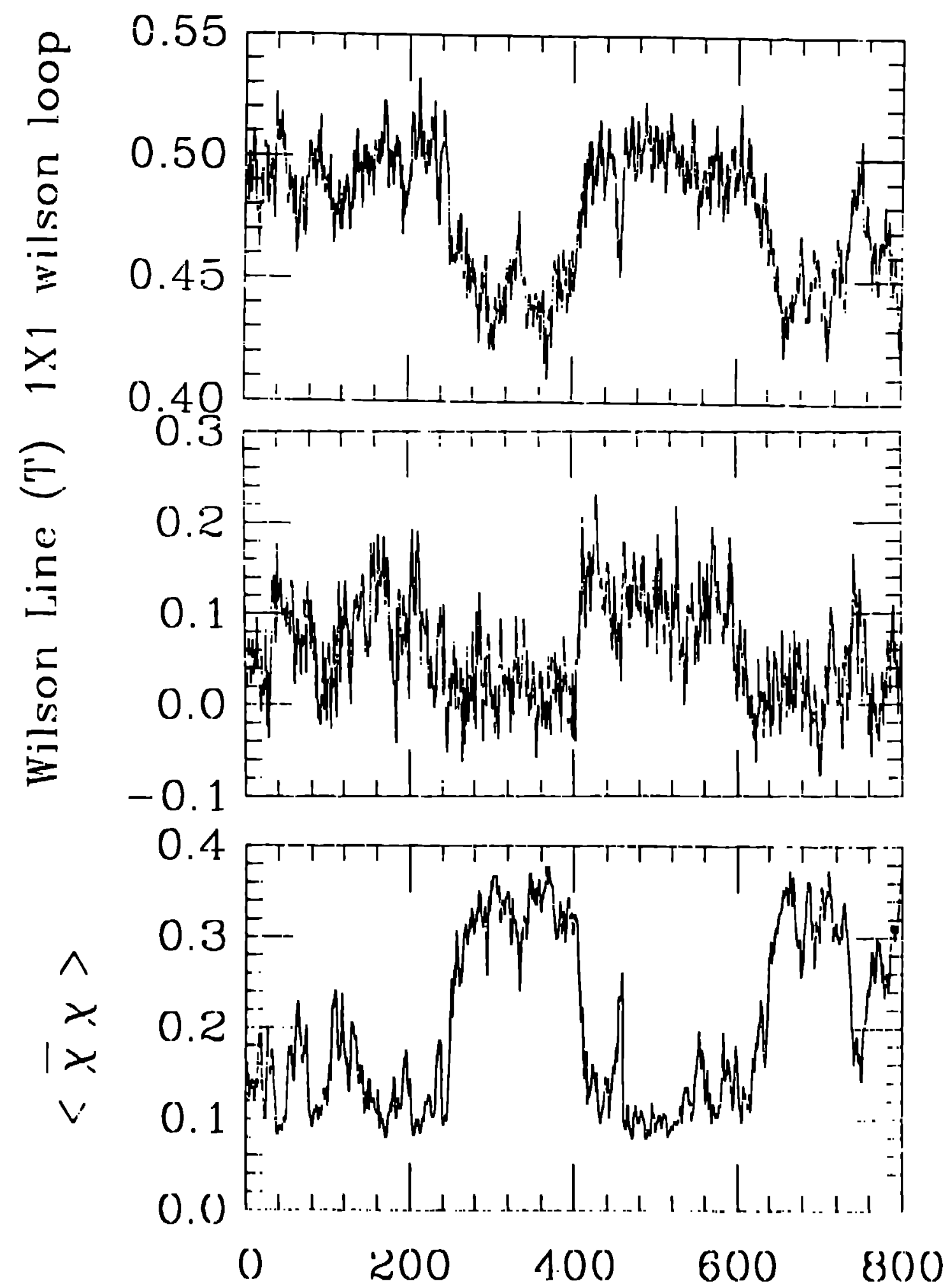

Fig.1 Plot of a) $1 \times 1$ Wilson loop, b) $\langle L\rangle_{\text {and }}$ ) $\bar{\chi} x$ with $\beta=4.95, m_{q}=0.05$ and $N_{c p}=60$ versus Monte Carlo Sweeps. 
rithm to some approximation, there can be a systematic bias. We discuss this later. In a Metropolis update, a link can be changed many times without having to recalculate $M^{-1}$. The multi-hit algorithm we use is that described in detail by Gavai and Gocksch [10]. We use antiperiodic boundary conditions in all directions. We update each link with $\mathbf{5 0}$ hits and the acceptance is adjusted to $\approx 30 \%$. In solving $A x_{\text {even }}=M^{\dagger} M x_{\text {even }}=b$, we define the convergence by $C=\frac{(b-A x|b-A I\rangle}{\langle x \mid x\rangle}$, which depends on the number of $C G$ iterations $\left(V_{c g}\right)$.

In our data all the observables, $\bar{\chi} \chi,\langle L\rangle$, Wilson loops and $R$ are correlated. In [1] we used $\bar{\chi} \chi$ to demonstrate that the transition for $m_{q}=$ 0.025 exists at $6 / g^{2}=4.9$. In Figs. 1 and 2 we show that with $N_{c g}=60$ there is a transition for both $m_{q}=0.05$ and $m_{q}=0.025$ at $6 / g^{2}=4.95$. The correlated flip-flops between the 2 states can be seen in $\bar{\chi} \chi,\langle L\rangle$ and in Wilson loops. So for $m_{q}=0.05$, the transition appears between $6 / g^{2}=4.9$ and 4.95 . Note that on increasing $6 / g^{2}$, the physical tomperature increases for fixed $N_{t}$ and so does the quark mass when the bare lattice quark mass is held fixed. Thus, for any given constant $m_{q}$, and $N_{t}$ we expect to go from the confined to the deconfined phase on increasing $6 / g^{2}$. This is what we see with the transition remaining strongly first order. We also find that the nature of the transition is unchanged under changing the 3 spatial boundary conditions to periodic as shown in Fig $2 c$.

To further analyze the transition we study $\bar{\chi} \chi$ as a function of $m_{q}$. The esiimates for $\bar{\chi} \chi$ in the two phases are shown in Fig. 3. In the confined phase we estimate $(\bar{\chi} x-0.3) \propto m_{q}$ from the data at $6 / g^{2}=4.9$. To study $\bar{\chi} \chi$ in the high temperature phase, we made runs with $N_{e g}=5 \mathrm{~J}$ at $6 / g^{2}=4.95$ and $m_{q}=0.05,0.025,0.02$ and 0.015 . We find a flip-lop at the two heavier $m_{q}$, while the system is predominately in the deconfined phitie at the two smaller $m_{q}$. The agreement with the expected behavior $\bar{\chi} \chi \propto m_{q}$ in the decnnfined phase is good. Unfortunately, this behavior though significant is not sufficient proof of the order of the transition. Thus a ccrraboration on lattices with larger $N_{t}$ and with $N \gg N_{t}$ is necessary. Further shortcomings of using Staggered fermions are that the flavor symmetry at finite lattice spacing is not $S U\left(n_{f}\right)$ and second the phenomenologically interesting case of 3 flavors is hard to achieve.

In [1] we presented an analysis of the systematic biases in our simuldtion. Our implementation of the CG algorithm tends to underestimnte the effects of the fermions, i.c. it tends to give too small a value for $S \equiv|\ln (\mathrm{R})|$. We have studied this by changing a single link and comparing the exact $R$ with that calculated with a variety of $C G$ sweeps. The exact $R$ is obtained by calculating the determinants, before and after changing the iink, using gaussian elimination. To study if there is an accumulation of the bias, we compare the product of the accepted determinant ratios $\left(A \equiv \ln R_{\text {ace }}\right)$ with 


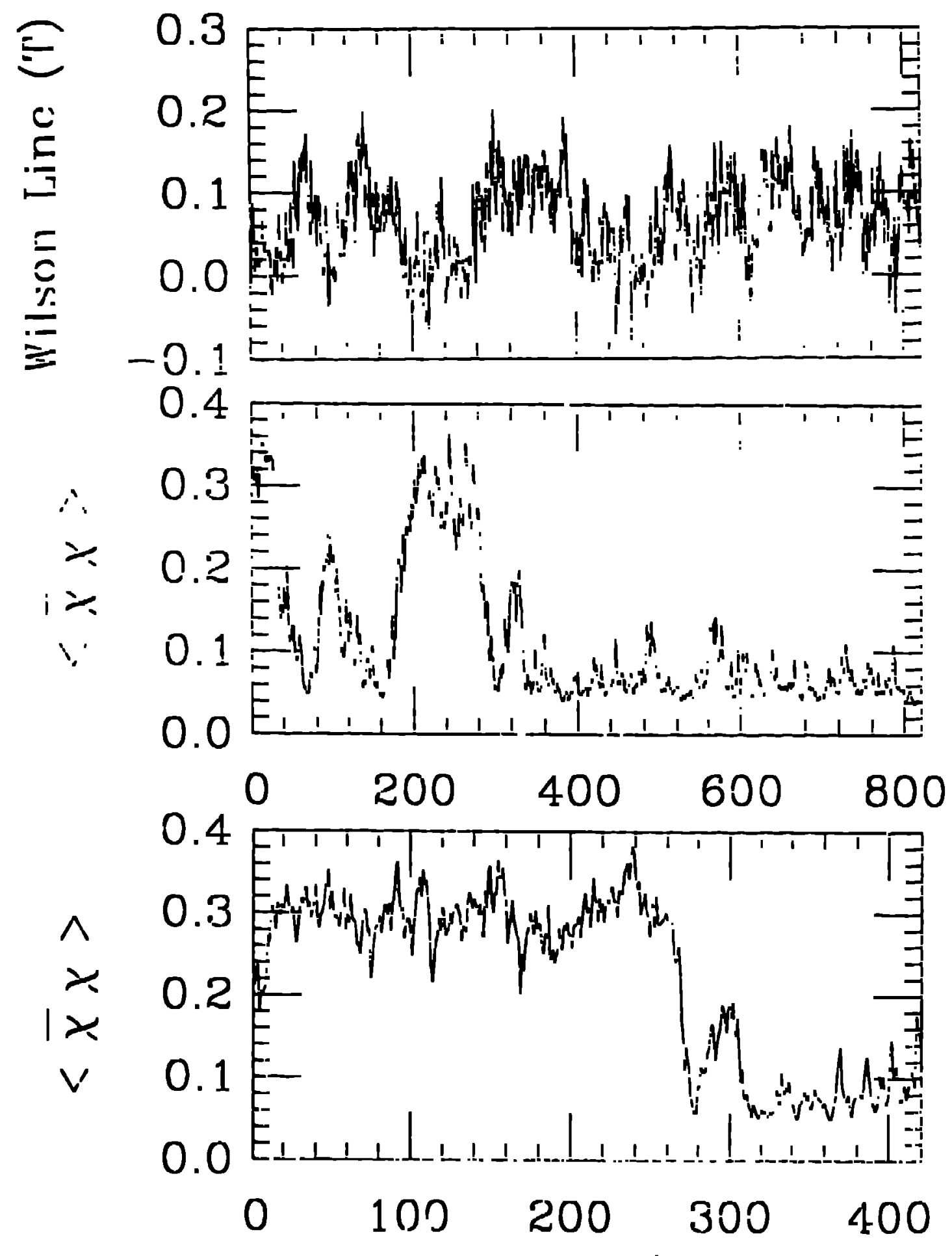

Fig.2 Plot of a) $(L), b) \bar{x} \times$ with anti-periodic b.c. In all directions and c) $\overline{X X}$ with periodic b.c. in opace - at $\beta=1.95, m_{q}=0.025$ and $N_{c o}=60$ versus Monte Carlo Swecps. 
the exact answer $(T)$.

The bias at $6 / g^{2}=4.95$ is similar to that at 4.9. The disagreement betiveen $A$ and $T$ gets progressively worse with decreasing $N_{E G}$, but $C$ is consistently a factor of $\approx 20$ smaller in the high temperature phase. Conversely, the bias decreases as $m_{q}$ increases. We again find that $C \leq 10^{-7}$ is necessary to avoid a bias at $m_{q}=0.025$ in both phases.

In Fig. 4 we show a distribution of the generated values of $R$ and those accepted in the Metropolis algorithm. We expect this distribution of $R$ to be a gaussian for a single link change in a fixed environment. That this is still approximately true when averaged over all links, as shown by the data, suggests that in a local update algorithm, the environment of any link is well described by a mean field approximation. Some properties of the distribution of the generated values of $R$ are; 1 ) the mean which is always less than one becomes smaller and 2 ) the width increases with decreasing quark mass and increasing $6 / g^{2}$. However, the change is small on going from $m_{q}=0.05$ to $m_{q}=0.015$. The relevance of this data is that for a method that gives an unbiased estimator for $R$ to be efficient, the spread of the estimate must be smaller than the narrow width shown.

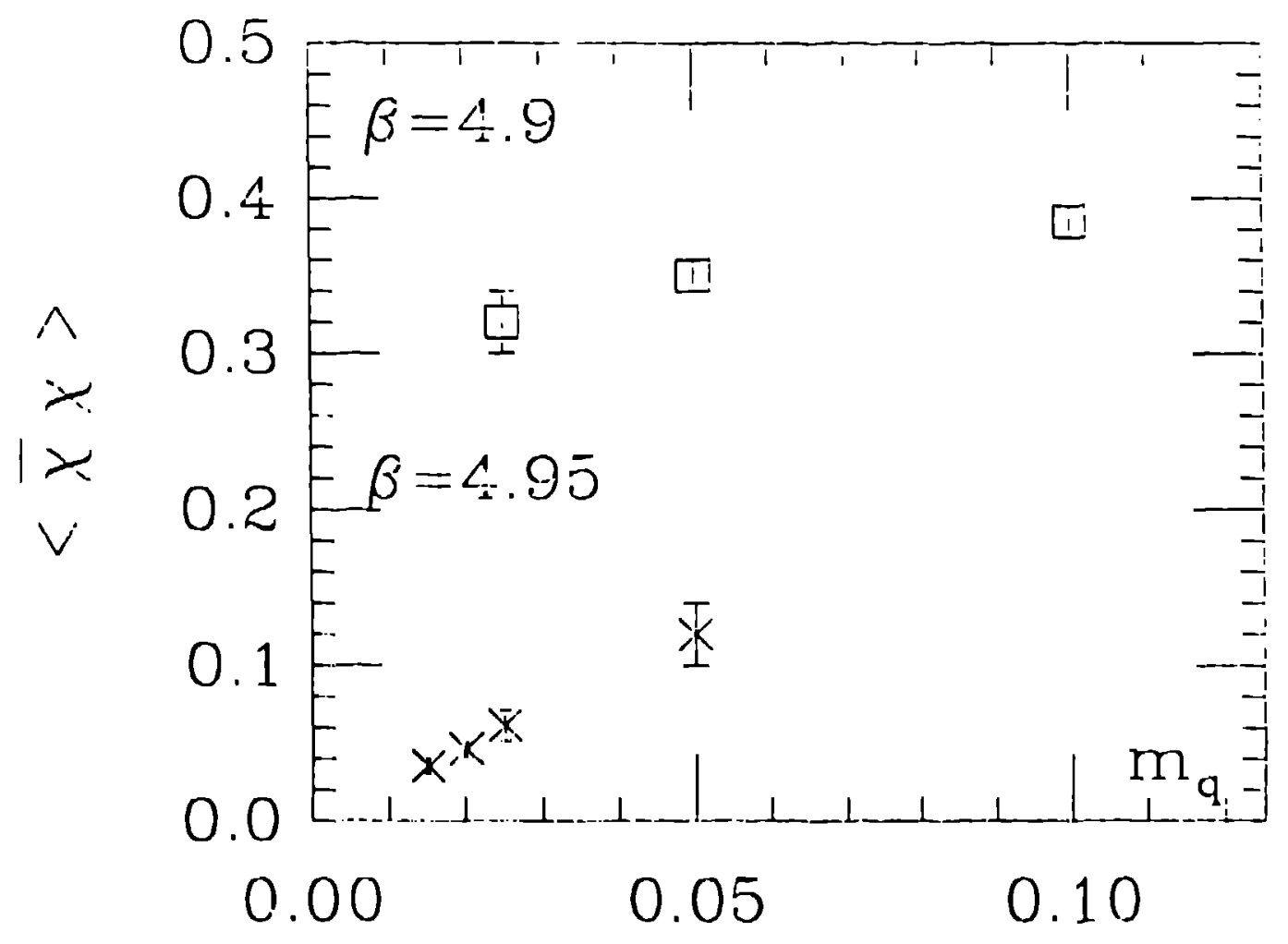

Fig.3 Plot of $\bar{x} x$ versus the bare quark rass 3 in lattice units. The expected chiral behavior is seen in both phases. 


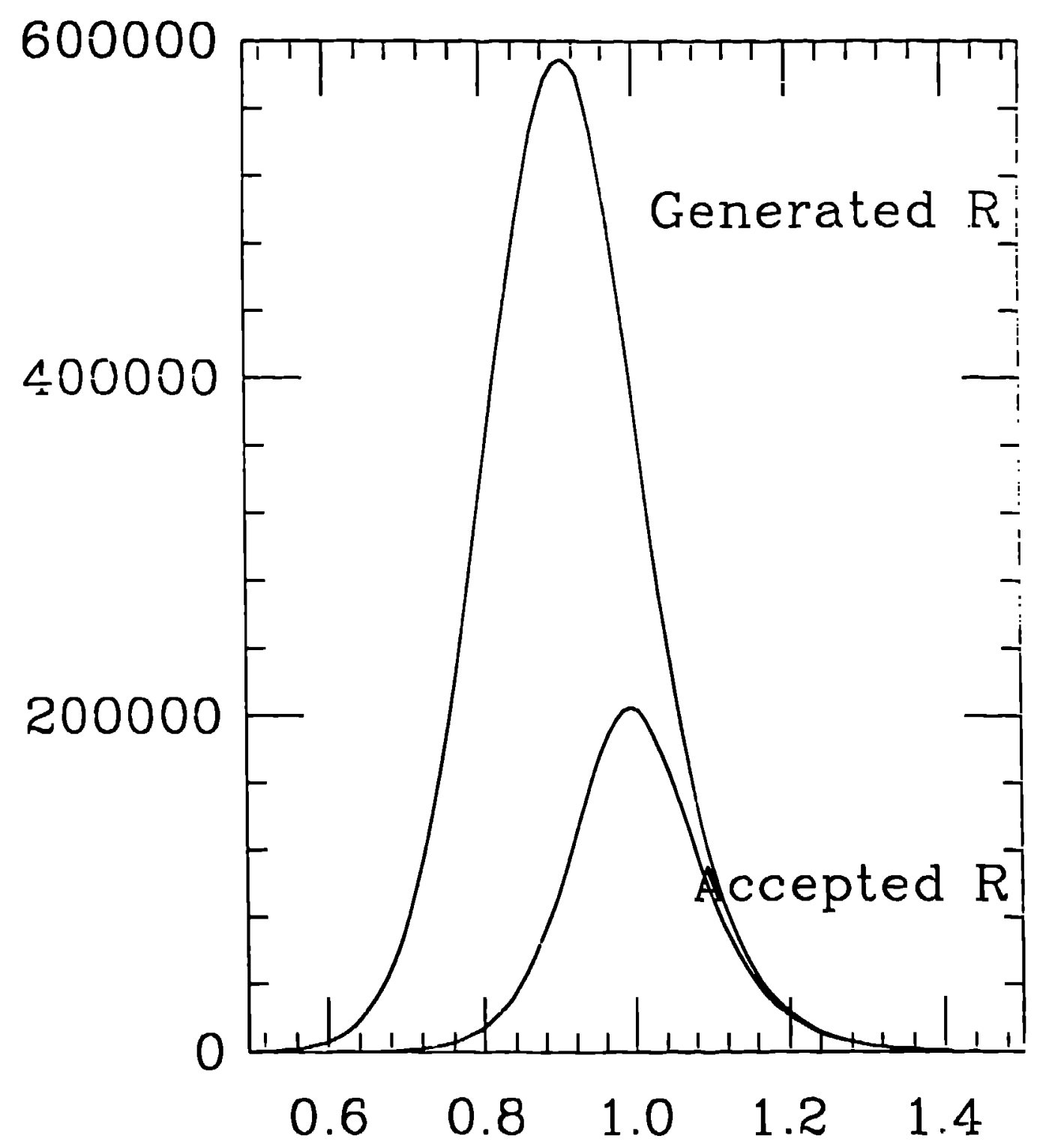

Fig.4 Distribution of the value of $\boldsymbol{R}$ generatel at each hit per link update. Also shown is the distribution of $\boldsymbol{R}$ for the hits accepted in the Metropolis algorithm. The data is at $\beta=4.95, m_{Q}=0.025$ and $N_{\text {cg }}=60$. 
To conclude, we present evidence that there does exist a first order chiral/thermal transition at small $m_{q}$. Since hadronic matter at high temperature and densily is expected to undergo a transition to quark-gluon plasma, which car. be checked by the planned heavy ion experiments, it is therefore very important to understand the nature of the transition and determine the transition temperature. Further, a first order transition in standard cosmology (thermal history) would dump entropy and affect the cosmological constant unless it occurs without appreciable supercooling. Thus one needs to know the transition rate. I hope these questions can be addressed by future lattice calculations.

\section{References}

[1] R. Gupta et. al. Rhys. Rev. Lett. 57 (1986) 2623.

[2] D. J. Scalapino and R. L. Sugar, Phys. Rev. Lett. 46 (1981) 519.

[3] J. B. Kogut, H. Mr.tsuoka, S. H. Shenktr, J. Shigemitsu, D. K. Sinclair, M. Stone and H. W. Wyld, Phys. Rev. Lett. 51 (1983) 869.

[4] R. D. Pisarsky and F. Wilr zek, Phys. Rev. I29 (1984) 338.

[5] B. Svetitsky, Proceedings of the Fifth International Conference on Ultra-Relativistic Nucleus-Nucleus Collisions, Quark Matter, 86 Asilomar; and M. Matsua, ibid.

[6] J. B. Kogut and D. K. Sinclair, Urbana Preprint ILL-(TH)-86-86-46.

[7] R. Gavai, Nucl. Phys. B360 (1986) 530.

(8) F. Fucito and S. Solomon, Phys. Rev. Lett. 55 (1985) 2641.

[9] M. Fukugita and A. Ukawa, Phys. Rev. Lett. 57 (1986) 503.

[10] R. Gavai and A. Gocksch, Phys. Rev. Lett. 56 (1986) 2659. 